\title{
Cardiac bypass pump flow management via NIRS monitoring
}

\author{
Andrew J. Macnab*, Roy E. Gagnon, Faith A. Gagnon, Derek Blackstock and \\ Jacques G. LeBlanc \\ Department of Pediatrics, Anesthesia and Surgery, Children's \& Women's Health Centre, University of \\ British Columbia, Vancouver, BC, Canada
}

\begin{abstract}
During cardiac surgery, bypass pumps rely on pressure monitors to evaluate flow. We studied whether it would be possible to optimize pump flow by monitoring changes in cerebral cytochrome $a, a_{3}$ using NIRS to maintain cyt redox status at its pre-bypass level. Method: 18 healthy $7-45 \mathrm{~kg}$ swine were placed on bypass for repeated cycles of cooling and re-warming from 36 to 15 to $36^{\circ} \mathrm{C}$ in $3^{\circ} \mathrm{C}$ steps. Between each cycle, the swine's bypass pump blood flow rate was adjusted to restore cytochrome redox status to its pre-bypass value. Results: In all swine trials, the number of pump flow alterations imposed by NIRS monitoring ranged from 0 to 42 , the average being 14 per trial. The best trial had 22 pump flow adjustments during which the range of cytochrome redox status change was $0.50 \pm 0.06 \mu \mathrm{moll}^{-1}$. The average trial had a range of cytochrome redox status change of $1.50 \pm 0.22 \mu \mathrm{mol}^{-1}$. Conclusion: NIRS-driven alterations in pump flow rate to maintain pre-bypass cytochrome redox status can be achieved successfully in the animal model.
\end{abstract}

Keywords: Spectrophotometry, nasopharyngeal, cytochrome, NIRO-300, oxygenation status

\section{Introduction}

Near infrared spectrophotometry (NIRS) has been used in the cardiac surgery setting for the past decade to detect adverse events, record patterns of change in oxygenation status, and optimize anaesthetic brain protection protocols $[3,8,10,13]$. The instrumentation typically includes 4 sequentially pulsed laser diodes emitting 777-911 nm wavelengths to the skin surface via a fiber optic bundle, and light detection either by photodiode arrays mounted on the skin's surface or by fiber optic transmission to a photon counting tube.

Clinically, NIRS is useful in measuring patterns of change in, the concentrations of oxygenated hemoglobin $\left(\mathrm{HbO}_{2}\right)$ and de-oxygenated hemoglobin $(\mathrm{Hb})$, as well as, in the redox status of cytochrome $a, a_{3}(\mathrm{Cyt})$. The $\mathrm{Hb}$ and $\mathrm{HbO}_{2}$ concentrations reflect delivery of oxygen to the tissues via the blood stream while the Cyt redox indicates oxygen utilization within the tissue cells. The Cyt molecule resides on the cristae of the intracellular mitochondria. It has a mass of 160 to 200 kilo-Daltons and contains two iron ions (cytochrome $a\left(\mathrm{Fe}_{\mathrm{a}}\right)$ and cytochrome $\left.a_{3}\left(\mathrm{Fe}_{\mathrm{a} 3}\right)\right)$, two copper ions $(\mathrm{CuA}$ and $\mathrm{CuB})$, a zinc ion and a magnesium ion. Single electrons are passed from $\mathrm{CuA}$ to $\mathrm{Fe}_{\mathrm{a}}$ and then to the $\mathrm{Fe}_{\mathrm{a} 3}-\mathrm{CuB}$ dimer where electron pairs are accumulated to stepwise reduce $\mathrm{O}_{2}$ in forming $\mathrm{H}_{2} \mathrm{O}[12,14]$. Prior to reduction, once sufficient electrons have accumulated, 4 pairs of protons enter the complex via the mitochondrion's low $\mathrm{pH}$ matrix zone [7,9]. The 4 protons not used in forming $\mathrm{H}_{2} \mathrm{O}$ exit to the high $\mathrm{pH}$ matrix zone (i.e., proton pumping) where 9 to 12 protons return to the low $\mathrm{pH}$ zone via the adinosine tri-phosphate (ATP)

\footnotetext{
*Corresponding author: Prof. A.J. Macnab, Critical Care Research Office, Room L317, Mail Box 80, Children's \& Women's Health Centre of BC, 4480 Oak St., Vancouver, BC, Canada V6H 3V4, Tel.: +1 604875 3524; Fax: +1 604875 2728; E-mail: amacnab@cw.bc.ca.
} 
synthase molecule causing that molecule to rotate thereby synthesizing ATP [11]. If oxygen availability, electron transport, and/or proton pumping is impaired, ATP synthesis will become impaired, and in turn cell metabolism will be compromised [4]. Cytochrome $a, a_{3}$ is the terminal enzyme in the respiratory chain and as a catalyst, its redox status reflects mitochondrial oxygen supply and demand.

During cardiopulmonary bypass surgery, adequate organ perfusion must be maintained, and perfusion of the brain is critical. The higher energy requirements of the brain mean that damage occurs quickly when oxygen and essential energy substrates are lacking. Cerebral hypoxic-ischaemic damage is irreversible. Several protocols have been developed to minimize or meet the brain's metabolic demands during cardiopulmonary bypass, including hypothermia and use of pharmacologic substances to reduce the brain's energy demand (e.g., Propofol) [8]. However, one of the most critical factors, the blood flow rate generated by the perfusion pump, is still determined empirically, and is followed predominantly by monitoring pressure.

In this experiment, we hypothesized that the pre-operative status of an animal's cerebral metabolism, as measured by NIRS, could be maintained by adjusting pump flow rate to increase or decrease $\mathrm{HbO}_{2}$ delivery in response to shifts in the Cyt equilibrium detected while on bypass.

\section{Method}

The subjects in the study were 18 healthy commercial swine (University of British Columbia Animal Care Certificate \#A01-0149). There were 3 animals in each weight group of 7, 10, 15, 20,30, and $45 \mathrm{~kg}$. Each animal was sedated with ketamine hydrochloride $(20 \mathrm{mg} / \mathrm{kg})$, anaesthesia was induced with isoflurane by mask. The animals were then intubated and maintained on isoflurane (2\%) throughout the remainder of the experiment. The subjects received intermittent positive-pressure ventilation at 10-30 breaths per minute, $50 \%$ inspiratory/expiratory ratio, $10-12 \mathrm{ml} / \mathrm{kg}$ tidal volume, and 0.60 fraction of inspired oxygen. Sensors were applied for continuous monitoring of heart rate, blood pressure, capillary oxygen saturation, nasopharyngeal temperature, and electrocardiology. Throughout the experiment blood samples were drawn for measurement of sodium, potassium, haematocrit, glucose, $\mathrm{pH}, \mathrm{pCO}_{2}, \mathrm{pO}_{2}$, base excess, $\mathrm{HCO}_{3}$ and oxygen saturation.

The NIRS photodiode array receiver for a NIRO-300 spectrophotometer (Hamamatsu Photonics KK, Hamamatsu City, Japan) was positioned on the intact skin surface of the brow $28 \mathrm{~mm}$ left of the longitudinal midline and along the lateral midline between eye and ear. The corresponding emitter optode was similarly placed along the lateral midline but $28 \mathrm{~mm}$ right of the longitudinal midline. The photodiode array was oriented directly at the emitter. The entire head was covered with a light-occluding drape.

Surgical preparation included exposing a femoral artery to install a blood inflow cannula from the cardiac bypass machine, and exposing the heart to install in the superior vena cava, an outflow cannula to return blood to the cardiac bypass machine. Once cannulation was complete, the blood circuit lines to the bypass machine were opened with initial pump flow set at three times the animal's body surface area (BSA) as determined by the DuBois equation:

$$
\text { BSA }=\text { weight }^{0.425} \cdot \text { length }^{0.725} \cdot 0.007184,
$$

where weight is in kilograms and length is in centimeters.

The bypass blood lines passed through a water heating/cooling jacket that was set to enable lowering the animal's core temperature in $3^{\circ} \mathrm{C}$ steps from $36^{\circ} \mathrm{C}$ to $15^{\circ} \mathrm{C}$ and then to return to normothermia in 
$3^{\circ} \mathrm{C}$ steps from $16.5^{\circ} \mathrm{C}$. At each temperature plateau the bypass machine's blood flow rate was adjusted, if necessary, to restore Cyt to its pre-bypass/post-cannulation value. The cycle of warming and cooling was repeated if theatre availability permitted.

NIRS data was collected throughout the experiment at a rate of $1 \mathrm{~Hz}$. Post collection analysis was done using Microsoft Excel 97 spreadsheet functions. Analysis of clinical NIRS data utilizes a modification of the conventional spectroscopic Beer-Lambert equation: $\log \left(I_{\mathrm{o}} / I\right)=\beta c t$ where $\beta$ is the reciprocal value of the thickness, $t$, in centimeters of a one molar solution, $c$ (concentration), when the light emerging from the solution, $I$, is one tenth of the original intensity, $I_{\mathrm{o}}$ entering it [15]. Since in clinical practice the in vivo sample volume is unknown, the thickness term, $t$, is modified to represent "pathlength" given as the separation between emitter and detector in centimeters multiplied by a tissue differential factor derived from a similar in vitro sample. Human differential pathlength factors (DPF) have ranged from 3.59 to 6.26 and can be estimated as for example: $\mathrm{DPF}_{\text {cranial }}=5.13+0.07 \cdot$ age $\mathrm{e}^{0.81}$ where "age" is between 1 and 50 years, the emitted light is $780 \mathrm{~nm}$ wavelength and the tissue is human cranial tissue [1,2]. At each wavelength the equation yields optical density values which must then be converted to chromophore concentration values. The common NIRS conversion algorithms employ matrix manipulations to derive simultaneous solutions for the unknown concentrations of the three principle chromophores of clinical importance $[5,6]$.

\section{Results}

In all trials, Cyt redox could be maintained at its pre-bypass status by pump flow adjustment.

The trial with the greatest change in Cyt redox status had a range of $2.4 \mu \mathrm{mol} 1^{-1}$ and had a standard deviation of $\pm 0.70 \mu \mathrm{mol}^{-1}$ with 42 pump flow changes (Fig. 1).

The trial with least change in Cyt redox status had a range of $0.5 \mu \mathrm{moll}^{-1}$ with a standard deviation of $\pm 0.05 \mu \mathrm{mol}^{-1}$ and had 22 bypass pump flow changes (Fig. 2).

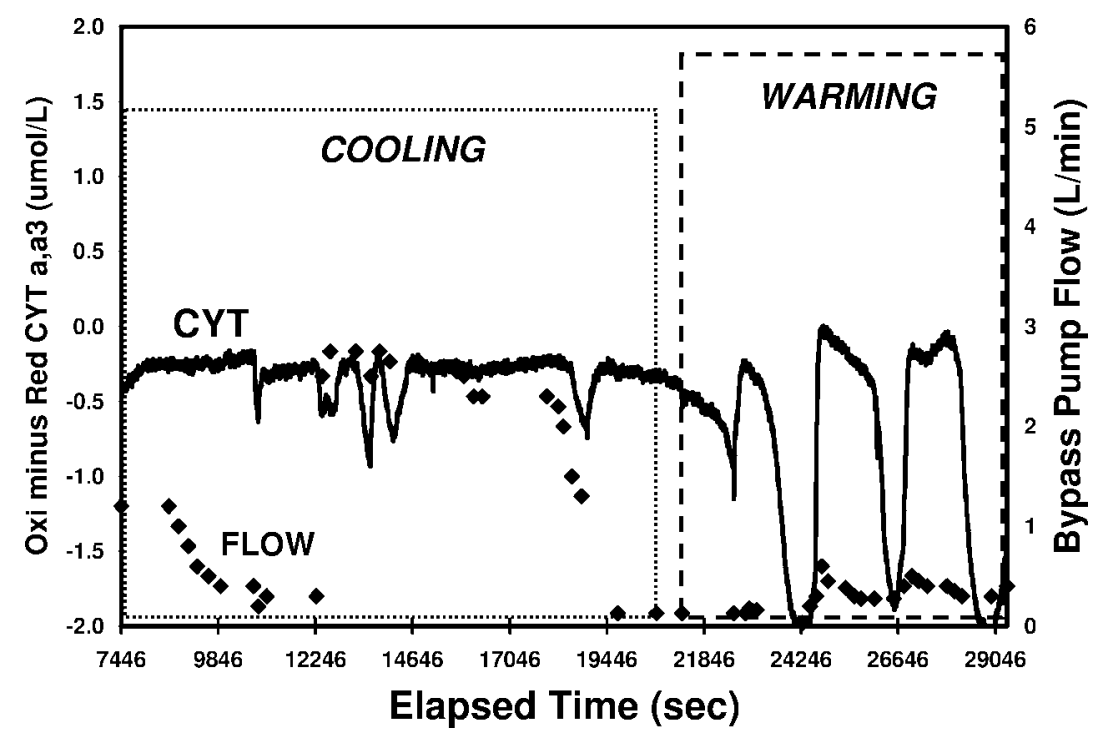

Fig. 1. The trial having the greatest changes in cytochrome $a, a_{3}$ redox status. 


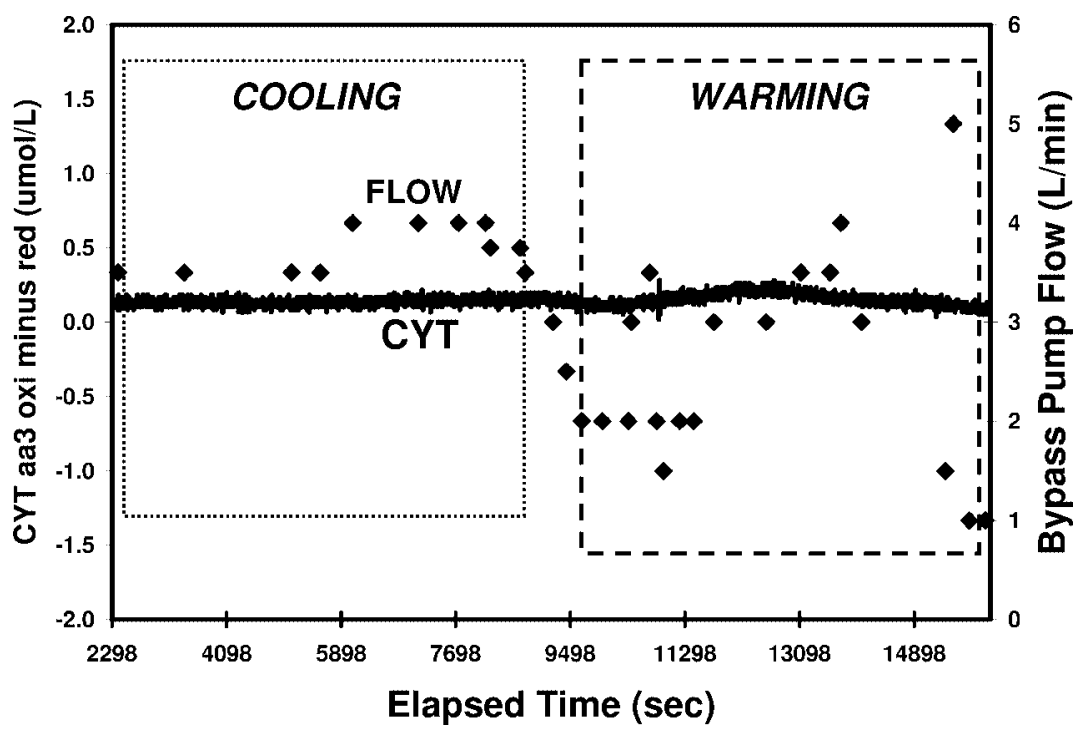

Fig. 2. The trial having the least change in cytochrome $a, a_{3}$ redox status.

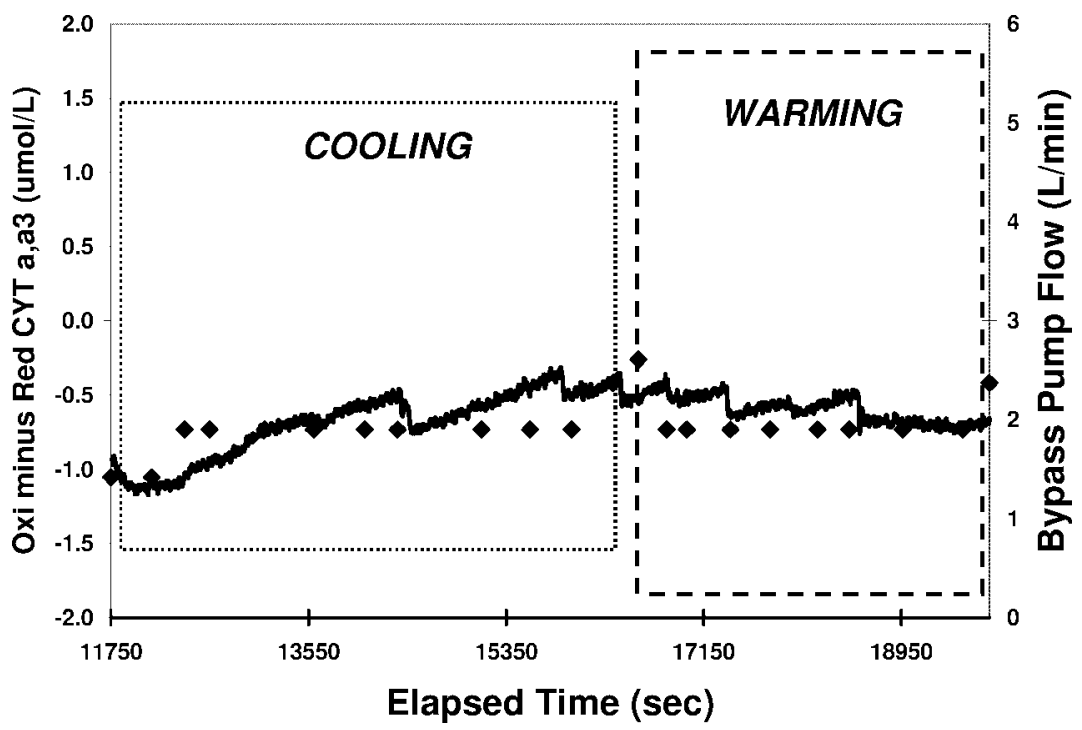

Fig. 3. A typical trial that closely matches the average change in cytochrome $a, a_{3}$ redox status for all 18 trials.

The average trial had a change in Cyt redox status of $1.5 \mu \mathrm{moll}^{-1}$, a standard deviation of $\pm 0.22 \mu \mathrm{moll}^{-1}$ with 14 pump flow changes (Fig. 3).

There was variation between individuals in how much change in pump blood flow rate was required and the required amount could not be predicted initially. Observation over at least two minutes was necessary to establish whether Cyt redox status would respond. This meant that there was a "learning" curve in the early stages of any trial. However, as a trial progressed, a greater certainty in the relationship between pump flow and redox status developed. Empirically, it seemed that a $0.11 \mathrm{~min}^{-1}$ alteration of pump blood flow rate would yield a $0.1 \mu \mathrm{moll}^{-1}$ change in Cyt redox status if the alteration(s) were 
being done continuously at the same pace as Cyt was changing. However, since the protocol had pump alterations occurring only at discreet steps, over-correction of pump flows sometimes occurred.

\section{Discussion}

This experiment demonstrates that there is a relationship between NIRS Cyt redox status and bypass pump flow rate, and that altering the flow rate delivered by the pump can counter any change in Cyt redox from its pre-bypass status. In some clinical settings, maintaining pre-bypass status may be critical while in others acquiring complete oxidation (or reduction) may be desirable. Since we have successfully maintained Cyt redox status via pump manipulations this technique should be adaptable to diverse clinical settings.

There is variation between individuals in the pump adjustment required. Estimating the amount of flow alteration required, and the direction of change requires experience. Making small flow changes gives a greater sense of control but may involve multiple adjustments to achieve sufficient change. Such adjustments take time that would prolong the duration of surgery and anaesthesia, which is undesirable. Alternatively, making large flow changes risks over-correcting redox status, which can also lead to extended time in counter corrections. In practice, there will probably be a degree of latitude to this process that will be physiologically acceptable, but we did not study this aspect. Ultimately, it would be desirable to have a nomogram for altering flow. In this study, we matched the exact pre-bypass cytochrome reading (to the nearest tenth of a $\mu \mathrm{mol}$ ) throughout the trial. In clinical practice, a less rigorous match between flow rate and redox status may be a physiologically acceptable compromise and would make pump flow adjustments in response to NIRS changes simpler to execute.

The effect on neurologic outcome of managing cardiac bypass pump flow rates via NIRS Cyt redox measurements has yet to be studied. Current pump management strategies rely upon pressure measurement ranges for a typical cohort. In contrast, NIRS has the advantage of monitoring a patient's specific cellular oxygenation status, so it is reasonable to expect that it could be a useful adjunct to current intraoperative protocols, and that neurological morbidity could be decreased as a result.

Other aspects of pump management requiring further Cyt studies are retrograde versus antegrade flows, startup haemodilution, startup volume loading, and the critical temperature range $\left(22-26^{\circ} \mathrm{C}\right)$. We do not believe any of these aspects will refute our findings here, but may help refine the methodology.

\section{Conclusion}

Cardiac bypass perfusion pump blood flow rates can be altered during cooling from and warming to normothermia to maintain near infrared spectrophotometer measurement of cytochrome $a, a_{3}$ redox at the pre-bypass status.

\section{Acknowledgement}

We wish to thank The Heart and Stroke Foundation of British Columbia and The Yukon for their financial support. 


\section{References}

[1] A. Duncan, J.H. Meek, M. Clemence, C.E. Elwell, P. Fallon, I. Tysszczuk, M. Cope and D.T. Delpy, Measurement of cranial optical pathlength as a function of age using phase resolved near infrared spectroscopy, Pediatr. Res. 39(5) (1995), 889-894.

[2] A. Duncan, J.H. Meek, M. Clemence, C.E. Elwell, I. Tsyzcruk, M. Cope and D.T. Delpy, Optical pathlength measurements on adult head, calf and forearm and the head of the newborn infant using phase resolved optical spectroscopy, Phys. Med. Biol. 40 (1995), 295-304.

[3] A.J. du Plessis, J. Newburger, R.A. Jonas, P. Hickey, H. Naruse, M. Tsuji, A. Walsh, G. Walter, D. Wypij and J.J. Volpe, Cerebral oxygen supply and utilization during infant cardiac surgery, Ann. Neurol. 37(4) (1995), 488-497.

[4] O. Farver, O. Einarsdottir and I. Pecht, Electron transfer rates and equilibrium within cytochrome c oxidase, Eur. J. Biochem. 267(4) (2000), 950-954.

[5] R.E. Gagnon, F.A. Gagnon and A.J. Macnab, Comparison of 13 published cytochrome c oxidase near-infrared spectroscopy algorithms, Eur. J. Appl. Physiol. 74(6) (1996), 487-495.

[6] R.E. Gagnon and A.J Macnab, C/C++ Coding for matrix pseudo inverses in clinical near infrared spectroscopy, Comput. Methods Biomech. Biomed. Engin. 1(2) (1998), 69-86.

[7] M. Karpefors, P. Adelroth, Y. Zhen, S. Ferguson-Miller and P. Brzezinski, Proton uptake controls electron transfer in cytochrome co oxidase, Proc. Natl. Acad. Sci. USA 95 (1998), 13 606-13 611.

[8] J.G. LeBlanc, D. Blackstock, A.J. Macnab, F.A. Gagnon, R.E. Gagnon, J. Russell and T. Ring, Effects of propofol on cerebral oxygenation during cardiopulmonary bypass in children, Can. J. Anesth. 47(11) (2000), 1082-1089.

[9] H. Michel, The mechanism of proton pumping by cytochrome c oxidase, Proc. Natl. Acad. Sci. USA 95 (1998), 12 81912824.

[10] G. Nollert, R.A. Jonas and B. Reichart, Optimizing cerebral oxygenation during cardiac surgery: a review of experimental and clinical investigations with near infrared spectrophotometry, Thorac Cardiovasc. Surg. 48(4) (2000), 247-253.

[11] G. Oster and H. Wang, Reverse engineering a protein: the mechanochemistry of ATP synthase, Biochim. Biophys. Acta 1458 (2000), 482-510.

[12] D.L. Rousseau, S. Singh, Y.C. Ching and M. Sassaroli, Nitrosyl cytochrome c oxidase. Formation and properties of mixed valence enzyme, J. Biol. Chem. 263(12) (1988), 5681-5685.

[13] M. Tamura, Non-invasive monitoring of brain oxygen metabolism during cardiopulmonary bypass by near infrared spectrophotometry, Jpn. Circ. J. 55 (1991), 330-335.

[14] C. Varotsis, Y. Zhang, E.H. Appelman and G.T. Babcock, Resolution of the reaction sequence during the reduction of $\mathrm{O}_{2}$ by cytochrome oxidase, Proc. Natl. Acad. Sci. USA 90 (1993), 237-241.

[15] A.I. Vogel, A Text-Book of Quantitative Inorganic Analysis Including Elementary Instrumental Analysis, 3rd edn, Longmans Green \& Co. London 1961, pp. 740-742. 


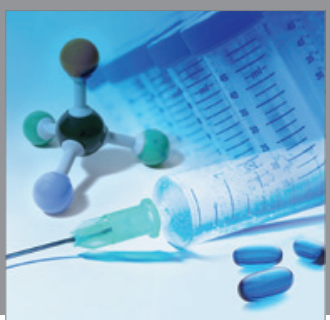

International Journal of

Medicinal Chemistry

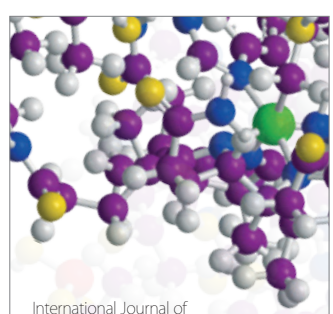

Carbohydrate Chemistry

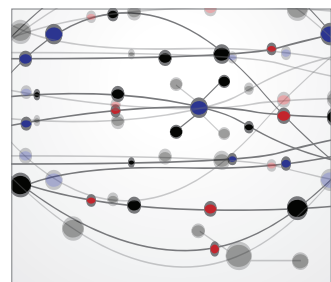

The Scientific World Journal
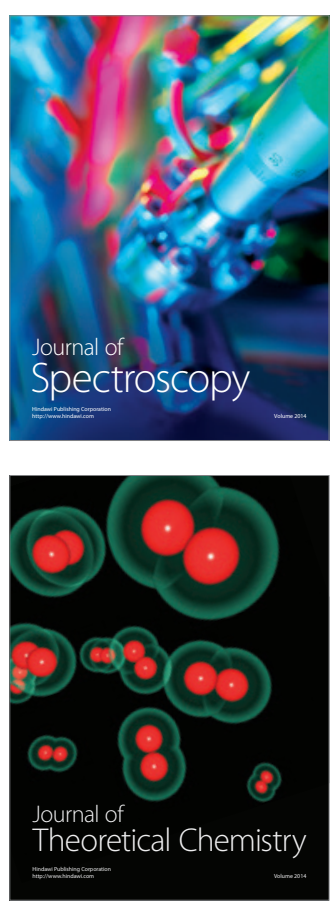
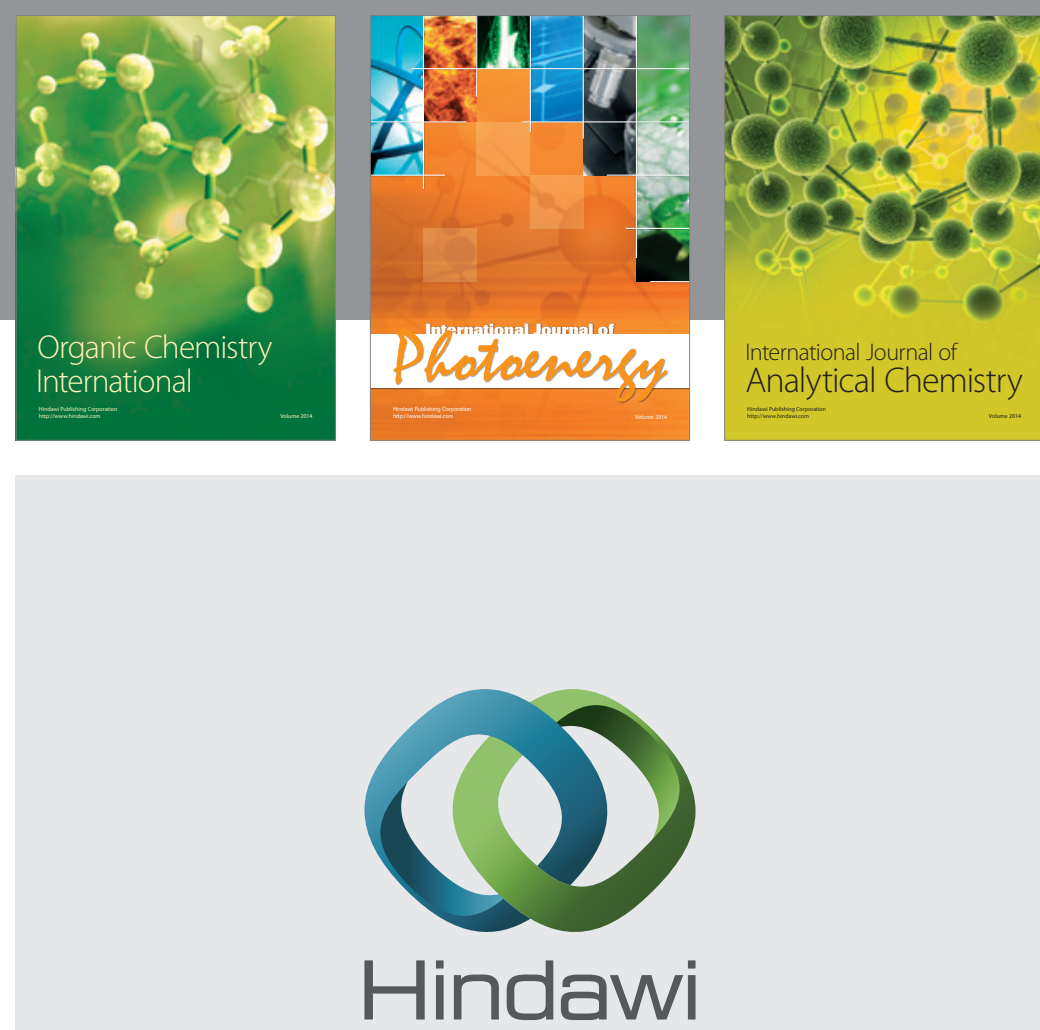

Submit your manuscripts at

http://www.hindawi.com
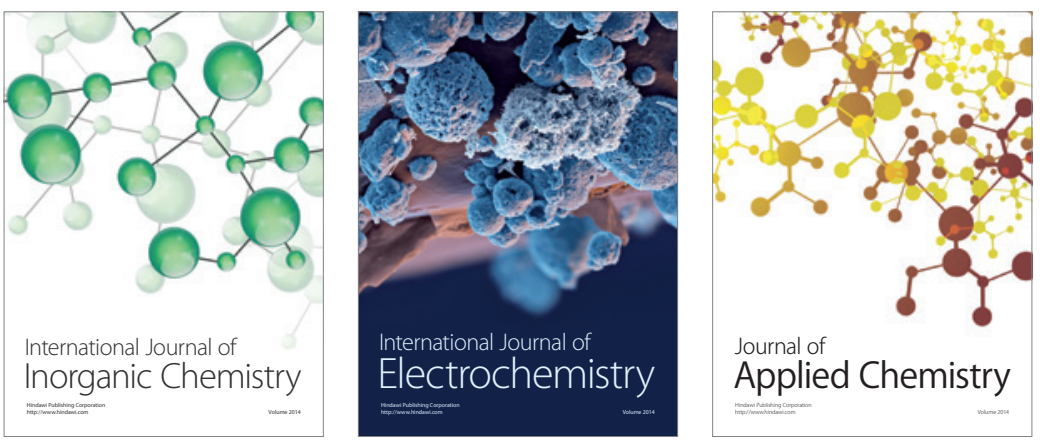

Journal of

Applied Chemistry
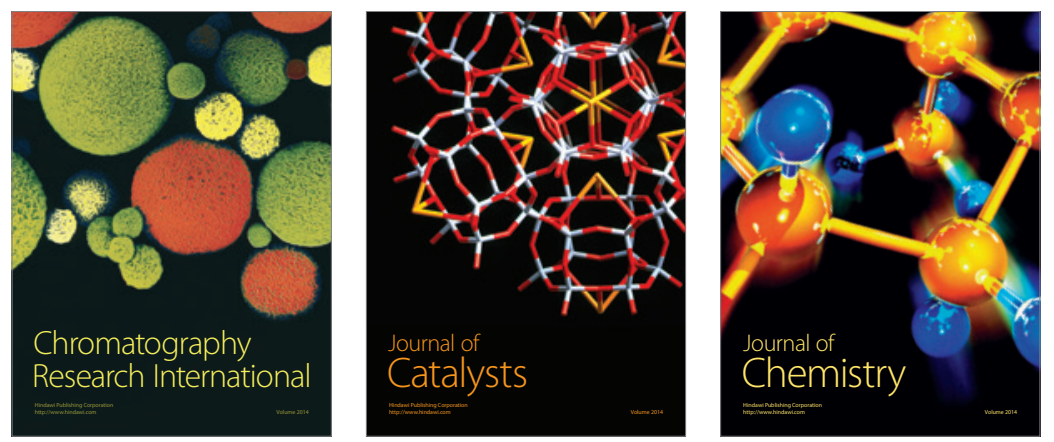
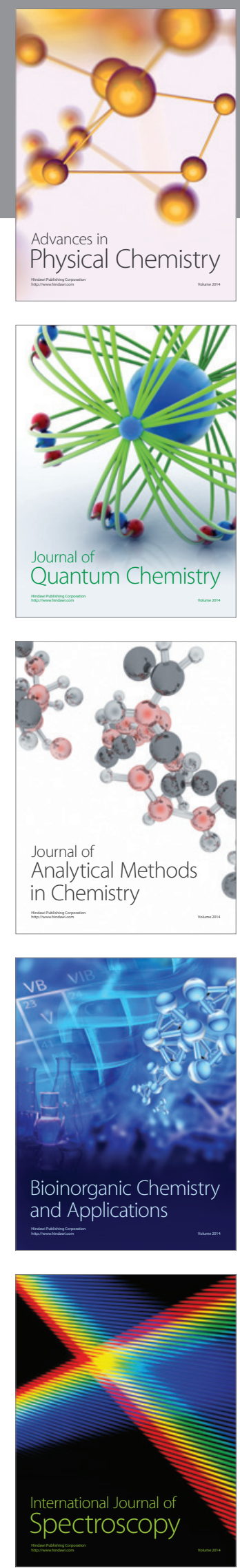